\title{
La lectura digital y la formación del lector digital en España: la actividad de la Fundación Germán Sánchez Ruipérez y el Proyecto Territorio Ebook'
}

\author{
Digital Reading and the Digital Reader Training in Spain: \\ The Germán Sánchez Ruipérez Foundation Activity and the Project \\ Territorio Ebook (E book Territory) \\ JOSÉ ANTONIO CORDÓN GARCÍA \\ Universidad de Salamanca \\ España \\ jcordon@usal.es
}

(Recibido: 25-11-2015; aceptado: 27-03-2016)

En marzo de 2016 se cumplieron 35 años desde la creación de la Fundación Germán Sánchez Ruipérez, una de las entidades que más han hecho por las bibliotecas y por la lectura en España. Lo importante de esta cifra es el testimonio que aporta acerca de la continuidad de una voluntad y un programa que se ha mantenido incólume a lo largo de los años, aportando uno de los raros ejemplos de supervivencia en un país donde las iniciativas culturales nacen con fecha de caducidad incorporada. Desde Álabe queremos rendirle homenaje a esta institución que ha sabido crecer con la sociedad a la que sirve.

\footnotetext{
${ }^{1}$ Para citar este artículo: Cordón García, José Antonio (2016). La lectura digital y la formación del lector digital en España: la actividad de la Fundación Germán Sánchez Ruipérez y el Proyecto Territorio Ebook. Alabe 13. [www.revistaalabe.com] DOI: 10.15645/Alabe2016.13.11
} 
Resumen. El desarrollo de las tecnologías de la información y la comunicación en los últimos cinco años ha provocado un cambio profundo en las prácticas de lectura y en los soportes en los que se efectúa esta, dando lugar a la aparición de una línea de investigación novedosa vinculada con los procesos implicados en la lectura digital. La Fundación Germán Sánchez Ruipérez, con el desarrollo de su pionero programa Territorio Ebook, ha representado un elemento clave para la exploración de este nuevo escenario. En el artículo se analizan algunos de sus principales resultados y se comparan con otras investigaciones desarrolladas en Europa principalmente.

Palabras clave: Lectura digital; libros electrónicos; Ipad; comprensión lectora; lectura social.
Abstract. The development of information and communications technology in the past five years has resulted in a profound change in reading practices and the media on which this takes place, leading to the emergence of a line of new research related to the processes involved in digital reading. The Germán Sánchez Ruipérez Foundation, with the development of its pioneering Ebook Territory program has been key to exploring this new scenario element. The article discusses some of the main findings and compares it with other research conducted mainly in Europe.

Keywords: Digital reading; ebooks; Ipad; reading comprehension; social reading. 


\section{Introducción}

Desde sus inicios la FGSR impulsa una intensa actividad en el desarrollo de un nuevo concepto de biblioteca, articulando un modelo abierto, dinámico, con espacios atractivos y sugerentes, al encuentro del usuario allí donde se encuentre (hospitales, escuelas, barrios, etc.), de un concepto de lectura activo, con la elaboración de un completo programa de promoción de la misma, de nuevos perfiles profesionales, con la configuración de un elenco de cursos, conferencias y encuentros especializados sin precedentes en España y, finalmente, de un programa de investigación fundamentado en tres factores determinantes para el mundo del libro en general y el de las bibliotecas y la lectura en particular: un centro de documentación modélico, unas colecciones de obras especializadas que durante muchos años constituyeron el referente de las publicaciones sobre el mundo del libro en el ámbito español y latinoamericano, y el diseño de un conjunto de investigaciones sobre el comportamiento de los distintos eslabones de la cadena de creación, distribución y recepción del libro que constituyen un referente a nivel internacional, con la traducción de las principales obras sobre el mundo de las bibliotecas y el libro existentes en el mundo y con la publicación de obras originales de los principales especialistas en la materia.

Estos antecedentes dan fe de una preocupación y una inquietud permanente por diagnosticar la situación de las bibliotecas, de la lectura y de los lectores y acometer soluciones que sirvan para situarlos en el momento actual, conscientes del papel fundamental que desempeñan las bibliotecas en las sociedades contemporáneas, de su importancia desde el punto de vista cultural, económico y social

\section{La lectura digital. Un cambio de tendencia en los patrones de comportamiento del público lector.}

En el informe sobre el libro digital en España publicado por El Observatorio de la Lectura y el Libro (2016) se pone de manifiesto el fenómeno, ya constatado en años anteriores, del crecimiento de la edición digital en nuestro país, al igual que ocurre en el resto de los países del mundo. Según éste el 72,5\% de editoriales publica actualmente libros electrónicos, cifra que es superior a la recogida en 2014, que llegaba solo al $68 \%$. Por otra parte, un $11 \%$ de las que no cuenta con ebooks planea integrarlos en su oferta en los próximos 2 años. Otro dato interesante, que da fe de esta progresión, es que el $62,1 \%$ de las editoriales señaló que tiene publicado menos del $50 \%$ de su catálogo. Las editoriales que ofrecen la totalidad de su catálogo en digital representan tan solo el 12,6\%. Según estos datos la progresión del sector en la hoja de ruta digital reviste aun un carácter ambivalente, en el que la voluntad de iniciar el recorrido por este nuevo entorno no siempre se concilia con los datos de la práctica editorial. 
De cualquier modo, durante estos últimos años, el ecosistema del libro ha experimentado un cambio radical en todos sus elementos, de tal manera que se cuestionan los eslabones tradicionales de la cadena editorial : la función del editor como intermediario y garante de la calidad formal y conceptual de los contenidos, articulador de colecciones que le confieran coherencia a una economía de prototipos, el papel de las librerías como núcleos de acceso al libro impreso, el papel de las bibliotecas como sitios de salvaguarda y categorización del saber, los derechos asociados a la función de autor y su dimensión económica, con la crisis del copyright y de la propiedad intelectual, la crisis de los intermediarios obligados a reinventarse e idear nuevas estructuras que les permitan sobrevivir en el contexto digital, en el que se está articulando una nueva cultura resultante de una economía del intercambio, de la colaboración, de la reputación, de la interactividad y de la integración, y en el que se ha producido la fractura digital con los nuevos lectores cada vez más acostumbrado al uso de la lectura electrónica. Una fractura digital que se manifiesta principalmente en el uso y popularidad de dispositivos de lectura nuevos, como smartphones y tablets, que favorecen el desarrollo de formas de escritura y lectura adaptadas a ellos

Las bibliotecas se encuentran en un momento de transición en el que las tecnologías de la información conforman realidades en permanente transformación (Alonso et al, 2016). La renovación tecnológica provoca una grave asincronía entre la evolución de la misma y su asimilación social. El libro se convierte en un territorio documental nuevo, en un lugar de encuentro de lectores y autores, donde los metadatos, los algoritmos de búsqueda y los sistemas de descubrimiento se erigen en plataformas de un nuevo ecosistema que tiene al lector como elemento central del mismo. El propio concepto de libro ha experimentado un cambio radical (Alonso; Cordon, 2015) en el que la importancia se desplaza del objeto al contenido, y aunque autores como Chartier (2006) o McKenzie (2005), así como otros estudiosos de la sociología de los textos, demostraran que las formas también producen sentido, este se va debilitando en beneficio de un contenido multiforme, ideado para un lector ubicado en un sistema tecnológico itinerante y permanentemente renovado, un lector ubicuo para el que la tecnología es cada vez más transparente y los conceptos que le afectan son los de accesibilidad, interoperabilidad y sociabilidad.

En este contexto, el desafío radicaba en analizar el proceso de transformación del lector en virtud de las nuevas características que se iban configurando en el acceso a los contenidos digitales, de las cuales han ido dando fe numerosos estudios y artículos en revistas especializadas internacionales, pero con poca incidencia en el caso español. Fue la Fundación Germán Sánchez Ruipérez una de las primeras instituciones en percibir la importancia del cambio que se estaba produciendo y en plantear iniciativas que analizaran su impacto y proyecciones futuras, tanto desde el punto de vista de la transformación del lector, como desde el de la necesaria adaptación de las bibliotecas al nuevo entorno que se estaba generando. 


\section{Un programa pionero en España y en Europa. Territorio Ebook.}

Cuando los libros electrónicos contaban con una escasa andadura, y una pequeña repercusión en el ámbito español, la Fundación Germán Sánchez Ruipérez lanza su programa Territorio Ebook, (2009) con la intención de analizar la incidencia de los nuevos soportes y dispositivos de lectura en el ámbito de las bibliotecas y de los usuarios de las mismas. Visto desde la distancia no deja de resultar sorprendente la precocidad de esta iniciativa, tanto a nivel nacional como internacional. Hay que tener en cuenta que las tablets todavía no existían, Apple lanzaría el Ipad en 2010, y los primeros dispositivos Android saldrían al mercado posteriormente. La tinta electrónica comenzaba su andadura comercial, con escasos dispositivos disponibles y a unos precios muy elevados. La oferta digital era casi inexistente, excepto unas pocas empresas lanzadas a la innovación y experimentación con nuevos soportes, el sector editorial aún no había percibido la importancia del cambio que se estaba produciendo a nivel global. En España el fenómeno de la lectura digital se había incorporado a los estudios que venía realizando el Ministerio de Cultura y la Federación de Gremios de Editores, a través de la publicación Hábitos de Lectura y Compra de Libros (hoy desgraciada e incomprensiblemente desaparecida después de dos décadas de andadura), sólo dos años antes, con el significativo epígrafe de $L a$ Lectura e Internet, pues lo singular del fenómeno estudiado era el hecho de que la gente utilizara la red para desarrollar actividades relacionadas con la lectura, contemplada como un ejercicio que se realizaba a través de la pantalla del ordenador. En 2009 solo un 10,1\% de los usuarios de Internet compraban libros a través de la red (13,3\% entre los lectores y un $2,8 \%$ entre los no lectores) no necesariamente digitales. Pero lo más sobresaliente es que, cuando se trataba de leer, más de un $80 \%$ leía directamente en pantalla y un $15 \%$ ¡!! los imprimía! En el informe publicado en 2011 sobre los hábitos de lectura del año 2010 se introduce ya el epígrafe sobre Lectura en soporte digital, incluyendo otros soportes distintos del ordenador. Pues bien, para ese año solo el 1,3\% de la población utiliza un e-reader para leer.

Estos datos pueden constituir una muestra de la arriesgada clarividencia de la FGSR para la puesta en marcha de un programa que apostaba por formas de lectura todavía experimentales y con escaso nivel de desarrollo, sometidas siempre a la contingencia del fracaso, como había ocurrido en los años de transición del siglo XX al XXI cuando tantas empresas que apostaron por lo digital acabaron feneciendo (Cordón; Gómez, Arévalo, 2011).

La idea de crear una etnografía de los lectores digitales que abarcara a todos los sectores de edad partía por lo tanto de una situación incierta, por las condiciones poco favorables para su desarrollo, y podía estar abocada a un intento más de creación de futuribles con escaso recorrido. Sin embargo, cinco años después del comienzo de este programa ya se puede valorar como uno de los grandes aciertos de la investigación sobre la migración digital en España. Territorio ebook nacía con tres 3 programas: Escuela (proyecto dedos) Biblioteca (5 investigaciones) y universidad (favorecer los encuentros, jornadas, etc.) Los 
fundamentos del mismo los podemos resumir en:

- Planificación modular adecuada, encarnada en la programación de las investigaciones según diferentes grupos de edad y diferentes dispositivos de lectura. Esto ha permitido analizar las prácticas de lectura de más de 300 lectores de entre 9 y 75 años, empleando para ello tanto lectores de tinta electrónica como Tablets.

- Incorporación paulatina de elementos de innovación tecnológica y social, como la incidencia que las redes sociales puedan tener en el desarrollo de conversaciones e iniciativas en torno a la lectura, articulando para ellos programas con el empleo de Twitter, Facebook y aplicaciones de lectura social como Readmill.

- Fuerte imbricación del binomio formación-investigación. Una de las aportaciones de este programa ha sido la demostración de que la formación constituía un elemento sustancial para el desarrollo de la lectura digital y que las variables relacionadas con la apropiación de los dispositivos y la comprensión lectora podían estar estrechamente relacionadas con la misma. La colaboración del personal de la FGSR y de diferentes grupos de investigación de la Universidad de Salamanca ha permitido conformar equipos de trabajo con el necesario contrapunto y complementariedad para la gestación de propuestas de trabajo innovadoras pero realistas. - Desarrollo de herramientas metodológicas y procedimentales adaptadas a los usuarios y los investigadores, según el contexto y los objetivo de cada investigación o acción formativa, estableciendo modelos extrapolables a otros contextos.

- Desarrollo de actividades de encuentro y dinamización de relaciones entre los actores implicados en el nuevo ecosistema digital.

\section{De la Piedra al Ebook}

Las investigaciones promovidas por la FGSR comenzaron en febrero de 2010 con el proyecto De la Piedra al Ebook. 40 lectores mayores de 55 años, socios de la Biblioteca Municipal de Peñaranda de Bracamonte, se enfrentaron a la obra Elmanuscrito de piedra de Luis García Jambrina, publicado por la editorial Alfaguara.

La obra se leyó en dispositivos de tinta electrónica por un grupo de edad ciertamente heterodoxo. 40 lectores mayores de 55 años se enfrentaban por primera vez en sus vidas con un sistema de lectura completamente nuevo. La singularidad de esta investigación radicaba no solo en los procedimientos, sino en la muestra, pues frente a las interpretaciones al uso que, infundadamente, como demostraron los resultados del estudio, presuponían que los nuevos entornos estaban reservados fundamentalmente para los calificados como Nativos Digitales, la FGSR asumió el reto de probar que los lectores digitales no nacen, sino que se hacen, estableciendo la formación como factor clave para su desarrollo. Esta hipótesis ha derivado, después de varios años de análisis, en un axioma incontrovertible, y en uno de los hallazgos de inevitable presencia en cualquier programa o política de promoción de la lectura digital que se quiera desarrollar. 
Otra de las particularidades de esta investigación, es el desarrollo de un conjunto de herramientas complementarias que permiten hacer un seguimiento de la misma e interactuar con los diferentes actores y protagonistas de esta. Al hilo de su lanzamiento se constituyó un Blog: De la Piedra al Ebook, centrado sobre el autor, la novela y los escenarios de la misma, concebido como punto de encuentro y dialogo entre los lectores y el ecritor v cu ohre
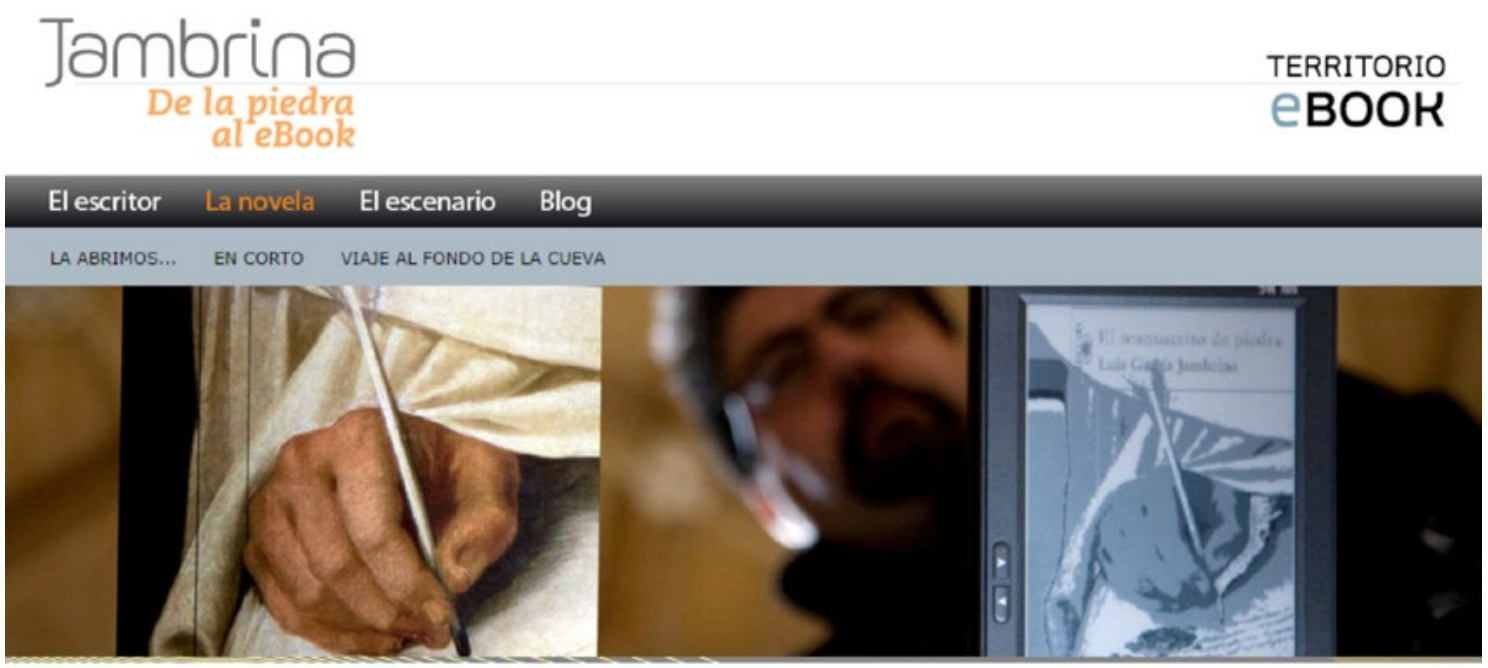

\section{La novela}
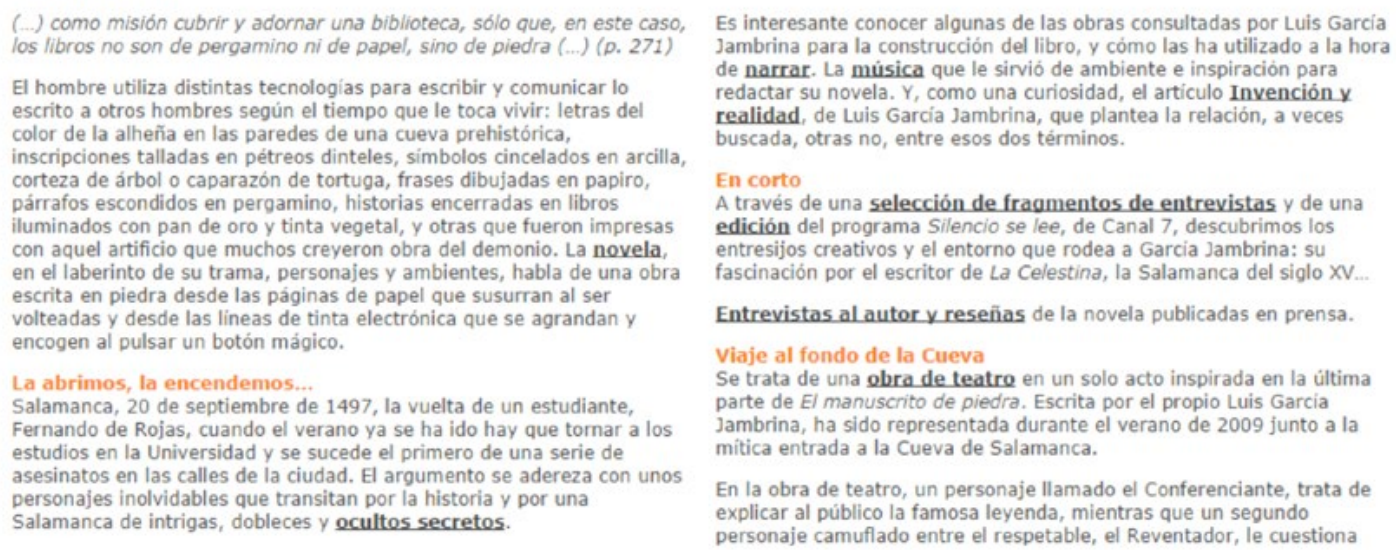

Es interesante conocer algunas de las obras consultadas por Luis Garcia Jambrina para la construcción del libro, y cómo las ha utilizado a la hora de narrar. La musica que le sirvió de ambiente e inspiración para redactar su novela. Y, como una curiosidad, el articulo Invención $x$ realidad, de Luis Garcia Jambrina, que plantea la relación, a veces

En corto

A través de una selección de fragmentos de entrevistas y de una edición del programa Silencio se lee, de Canal 7, descubrimos los entresijos creativas y el entorno que rodea a fascinación por el escritor de ta Celestina, la Salamanca del siolo XV.... Entrevistas al autor y reseñas de la novela publicadas en prensa.

Viaje al fondo de la Cueva

Se trata de una obra de teatro en un solo acto inspirada en la última parte de El manuscrito de piedra. Escrita por el propio Luis Garcia Jambrina, ha sido representada durante el verano de 2009 junto a la mitica entrada a la Cueva de Salamanca.

En la obra de teatro, un personaje llamado el Conferenciante, trata de explicar al público la famosa leyenda, mientras que un segundo personaje camuflado entre el respetable, el Reventador, le cuestiona

Página y blog De la piedra al ebook: http://www.territorioebook.com/jambrina/blog

Concluida la investigación los responsables de la misma afirman: Los mayores de 55 asumieron sin más no estar habituados a las nuevas tecnologías, eran inmigrantes, incluso analfabetos digitales o desconectados $y$, sin embargo, demostraron buena receptividad, no presentaron las resistencias por todos vaticinadas, buscaron más la eficacia lectora sobre el dispositivo que los aspectos más técnicos como la conectividad, se dejaron acompañar en la lectura y se decantaron sin lugar a dudas por un dispositivo sencillo, que les ofreciera algo que nunca habían imaginado. Son lectores digitales vírgenes, sin aspiraciones tecnológicas, que han alcanzado mucho más de lo que hubieran podido imaginar, 
y que han transitado del papel a lo electrónico de una manera asombrosamente natural. En realidad, no importa si leen en papel o en un dispositivo, lo que importa es el acompañamiento de la biblioteca. (Sánchez, Emilio et. al., 2011)

Es muy importante poder contextualizar el momento en el que se hace esta primera investigación. En ese año, y desde el punto de vista empresarial, la oferta de libros electrónicos en España es todavía pobre y muy fragmentada, con plataformas de creación reciente como Libranda, a iniciativa de importantes grupos editoriales como Planeta, Santillana, Ramdon House, Mondadori, asi como editoriales tradicionales que lanzan alguna colección en formato digital como la editorial catalana Grup 62 y el Institut Cambó, librerías digitales que comercializan obras de autores noveles o distribuyen títulos de editoriales que carecen de plataforma propia como Leqtor, sitios web personales de autores, que comercializan sus propias obras, y empresas de venta de dispositivos, que al hilo del éxito de los libros electrónicos y su tecnología asociada se lanzaron a la venta de contenidos on line, como leer-e o Luarna, y plataformas de venta y distribución de contenidos electrónicos como Todoebook.

Esta investigación, además, se hace en momento en el que en casi todos los países del mundo el fenómeno estaba siendo estudiado únicamente en los sitios en los que el libro electrónico había alcanzado una significación cuantitativamente importante: las universidades, pero apenas había penetrado en la población en general. Los estudios de usuarios eran escasos, o con muestras poco significativas a pesar de la repercusión mediática que pudieran representar sus conclusiones.

Los libros electrónicos representaban un área de estudio nueva para cuyo análisis se estaban empleando metodologías muy diferentes y áreas de aproximación muy diferenciadas. Los estudios más abundantes eran los que consideran los libros electrónicos en su contexto académico, como los desarrollados por Derrick Fernandez para la Universidad de Hillintong, Wilkins para la de Derby , McLuckie en Zuricht, Allen para la de Illinois o, con carácter más general, Bellaber y Gillette, Sckeker y McLellan, Nelson, o Anderson . Junto a estas se desarrollaron también encuestas sobre un universo más amplio de población como las realizadas por el IDPF, Springer, Publishers Communication Groups; Centre for Information Behaviour and the Evaluation of Research (CIBER), Joint Information Systems Committee (JISC), etc (Cordón, Gómez; Arévalo, 2011).

Las preocupaciones del momento no diferían mucho de las que se estaba planteando la FGSR con sus programas, y que David Stern (2010) resume con precisión. Plantea este autor una serie de cuestiones que era interesante conocer sobre el uso de los libros electrónicos: ¿Cómo se usan los libros electrónicos? ¿Qué supuestos fundamentan su aceptación? ¿Se utilizan como se hace con las versiones en papel o se aprovechan sus nuevas funcionalidades? ¿Qué opciones de diseño, navegación, búsqueda, etc., incorporan? ¿Constituyen una sustitución o un suplemento del material impreso?

En un estudio desarrollado por el JISC e-book observatory proyect, por esas fechas, en el que participaron más de 40.000 estudiantes, se obtuvieron interesantes ob- 
servaciones en relación con el uso que estos hacían de los libros electrónicos; entre ellas, que se utilizaban preferentemente para consulta, no para una lectura continuada, dado que un 70\% de los estudiantes hojeaban capítulos sueltos, que la ratio de uso estribaba en unos 13 minutos por sesión, unas 8 páginas de visionado, que los empleaban como referencia para completar datos aportados en las obras impresas, a las que acudían para una lectura más en profundidad y que los libros electrónicos no eran considerados como sustitutos de los libros impresos, por lo que su impacto en el mercado era nulo. Su uso era considerado más como un complemento que como una solución autónoma.

Por poner otro ejemplo de la situación contextual, en marzo de 2009 se realizó una encuesta por parte de The Cocktail Analysis, con objeto de comprobar la actitud de los usuarios hacia los dispositivos de lectura y los libros electrónicos. Según los resultados obtenidos, en el mercado español aún se estaba muy lejos de un conocimiento siquiera básico sobre el e-book y el impacto de éstos sobre los hábitos de lectura. Aunque el 75\% los ha escuchado nombrar, sólo 3 de cada 100 personas poseía uno (no incluyendo los smartphone). La gran mayoría de la gente no identificaba ninguna marca específica $(70,3 \%)$ aunque el Kindle de Amazon y el Ready de Sony eran los más conocidos con un $12,6 \%$ y $7,9 \%$ respectivamente.

Además, un elevado porcentaje de personas no estaban en absoluto interesadas en adquirir uno de estos dispositivos. La tasa de lectura disminuye según aumenta la edad. En 2010, los más jóvenes son los que presentan una tasa de lectores mayor (70,5\%). De los 25 a los 54 años la tasa de lectores se sitúa en valores próximos al 60\%; de los 55 a 64 años se aprecia el primer descenso brusco $(47,9 \%)$ y entre los mayores de 65 años vuelve a descender bruscamente hasta 29,8\%. (Hábitos de lectura, 2010)

La apuesta no podía ser más arriesgada al elegir a un grupo de edad que lee poco, en cualquier tipo de soporte, y que se muestra renuente al uso de las tecnologías.

\section{BeatlePad}

En octubre de 2010 se pone en marcha la segunda fase del proyecto Ebook y Biblioteca, con otra investigación, en este caso dirigida al estudio de los lectores comprendidos entre los 13 y los 18 años. La obra elegida para la investigación es Eljoven Lennon de Jordi Sierra i Fabra, publicado por la editorial SM.

En este caso la investigación da una vuelta de tuerca radical, proponiendo un experimento completamente innovador en el ámbito digital de cuyos resultados dependerían muchas de las prácticas que se pudieran articular posteriormente en las bibliotecas. La incorporación de una Tablet, el Ipad, como dispositivo de lectura, entraña un desafío importante por cuanto se trata de un aparato diseñado para el desarrollo de actividades no exclusivamente, ni principalmente, de lectura, como ocurría con los e-reader. Si en el caso de los dispositivos de tinta electrónica empleados en la fase anterior de las investigaciones se había recurrido a un aparato de carácter mimético, en tanto que reproducía el entorno analógico de una manera muy precisa, aunque con prestaciones añadidas, ahora 
se elige una tecnología todavía de escaso recorrido en el mercado, pues su presentación oficial tuvo lugar solo unos meses antes, y con funcionalidades entre las que la lectura no es la principal. Por otra parte, se trata de un dispositivo de pantalla retroiluminada, lo que podría condicionar sustancialmente la experiencia de lectura. El reto era ¿aceptaran los lectores de manera natural la lectura en un aparato "extraño", alejado completamente del entorno impreso? ¿Aprovecharan las funcionalidades del mismo para enriquecer su experiencia de lectura? En definitiva, se trataba de averiguar los niveles de apropiación de un dispositivo nuevo desde todos los puntos de vista, en su primera versión, además, que sería mejorada considerablemente en años posteriores.

El experimento podría constituir un elemento de análisis y debate importante. No hay que olvidar que un año antes se había publicado en España el libro de Gary Small El cerebro digital: como las nuevas tecnologías están cambiando nuestra mente (Urano, 2009) en el que se platea la incidencia negativa que Internet y las funcionalidades asociadas pueden tener sobre la sociedad, modificando sus formas perceptivas y sensitivas. En la misma línea que Nicholas Carr argumentaría posteriormente (2010) señalando que:

Durante los últimos años he tenido la sensación incómoda de que alguien, o algo, ha estado trasteando en mi cerebro, rediseñando el circuito neuronal, reprogramando la memoria. Mi mente no se está yendo - al menos, que yo sepa-, pero está cambiando. No pienso de la forma que solía pensar. Lo siento con mayor fuerza cuando leo. Solía ser muy fácil que me sumergiera en un libro o un artículo largo. Mi mente quedaba atrapada en los recursos de la narrativa o los giros del argumento, y estaría horas surcando vastas extensiones de prosa. Eso ocurre pocas veces hoy. Ahora mi concentración empieza a disiparse después de una página o dos. Pierdo el sosiego y el hilo, empiezo a pensar quéotra cosa hacer. Tesis que se verían reforzadas años más tarde en otra de sus obras Atrapados: como las maquinas se apoderan de nuestras vidas (Taurus, 2014).

Por lo tanto, enfrentar a 40 adolescentes y jóvenes, menores de 18 años a este soporte de lectura constituiría una experiencia única de afirmación o refutación de las hipótesis que se estaban gestando sobre lo digital, con grandes repercusiones mediáticas.

Por otra parte, la obra elegida constituía igualmente una invitación al descubrimiento o al rechazo. ¿en qué medida un grupo de lectores comprendidos entre los 13 y los 18 años, nacidos entre 1993 y 1997 comprenderían la vida y hechos de un músico cuyas obras se habían desarrollado en la década de los 60 y principios de los 70, 30 años antes de que ellos nacieran? Es cierto que las obras de los Beatles estaban experimentando un revival espectacular con la publicación de grabaciones inéditas, y que el nombre de Jordi Sierra i Fabra constituía un elemento de refuerzo importante para un público lector familiarizado con otro tipo de personajes. El contenido de la obra, independientemente del nivel de conocimiento del personaje, también está pensado para despertar la curiosidad de cualquier adolescente. Un joven de 15 años, que vive en Liverpool, que frecuenta el puerto de su ciudad donde conoce a marineros que traen discos de un nuevo 
fenómeno llamado rock and roll. A partir de ahí crea su propio conjunto y aprende a tocar la guitarra de forma autodidacta. Un día conoce a otro entusiasta como el, Paul McCartney, con quien simpatiza y le ofrece entrar en su grupo. Poco después conocen a George Harrison, que sólo tiene trece años, pero toca muy bien la guitarra. John se muestra reticente a su entrada, pero su calidad vence los reparos de la edad y se crea el germen de los Beatles. En fin, una historia muy similar a la de los cientos, o miles de grupos que se han ido formando en el mundo, con diversa fortuna, pero que en este caso dieron lugar a uno de los fenómenos culturales y mediáticos más importantes del siglo XX.

La investigación, como se ha destacado más arriba, se desarrolla en un momento en el que los contenidos digitales van adquiriendo importancia, aunque aún revisten una presencia puramente testimonial en el conjunto de la producción editorial.

Si observamos los datos del Comercio Interior del Libro, para el año 2010, podemos comprobar el contexto real de la producción electrónica. Una circunstancia que sobresale es que la cifra de libros electrónicos es inferior a la de los títulos impresos transformados a soporte digital, lo que apunta a una actitud sumamente conservadora por parte de los editores, que apuestan por valores seguros antes que acometer empresas de resultado incierto. Esto explica igualmente que las obras destinadas a nuevos dispositivos de lectura como las tablets tengan una presencia insignificante (un $2,8 \%$ del total), y que los ordenadores sigan constituyendo el soporte de destino preferente para la producción digital. El hecho de que la mayoría de las obras se publiquen en pdf, no hace sino alimentar esta circunstancia. Se opera con formatos con escasas posibilidades de intervención por parte de los lectores, con fotos fijas de obras que garantizan la fidelidad a un original impreso, seguramente en la creencia de que la transición digital pasa por la facilidad de identificación, por parte del lector, de la forma y contenido de sus experiencias precedentes.

Todo este contexto marca unos límites y posibilidades a las metodologías y circunstancias en las que se puede desarrollar una investigación sobre la lectura digital, pues si el nuevo entorno facilita determinadas prestaciones de lectura como las relacionadas con la interactividad y los contenidos enriquecidos, esto solo será posible en la medida en la que los productores de los mismos los codifiquen con estas funcionalidades.

En el caso de El Joven Lennon, la obra se publicó por primera vez en el año 1988, por ediciones SM, y fue objeto de dos ediciones más, la última en el año 2005. En todo caso, cuando se publica la obra en 1988, los ordenadores acababan de desembarcar en el ámbito doméstico y la lectura a través de los mismos carecía de tradición.

No fue hasta 20 años después de la publicación de la novela cuando esta contó con una página propia en Facebook, con comentarios de los lectores, pensamientos de John Lennon y noticias diversas acerca de su vida y su obra, posterior al desarrollo de la investigación, pues se pone en marcha en noviembre de 2011. 


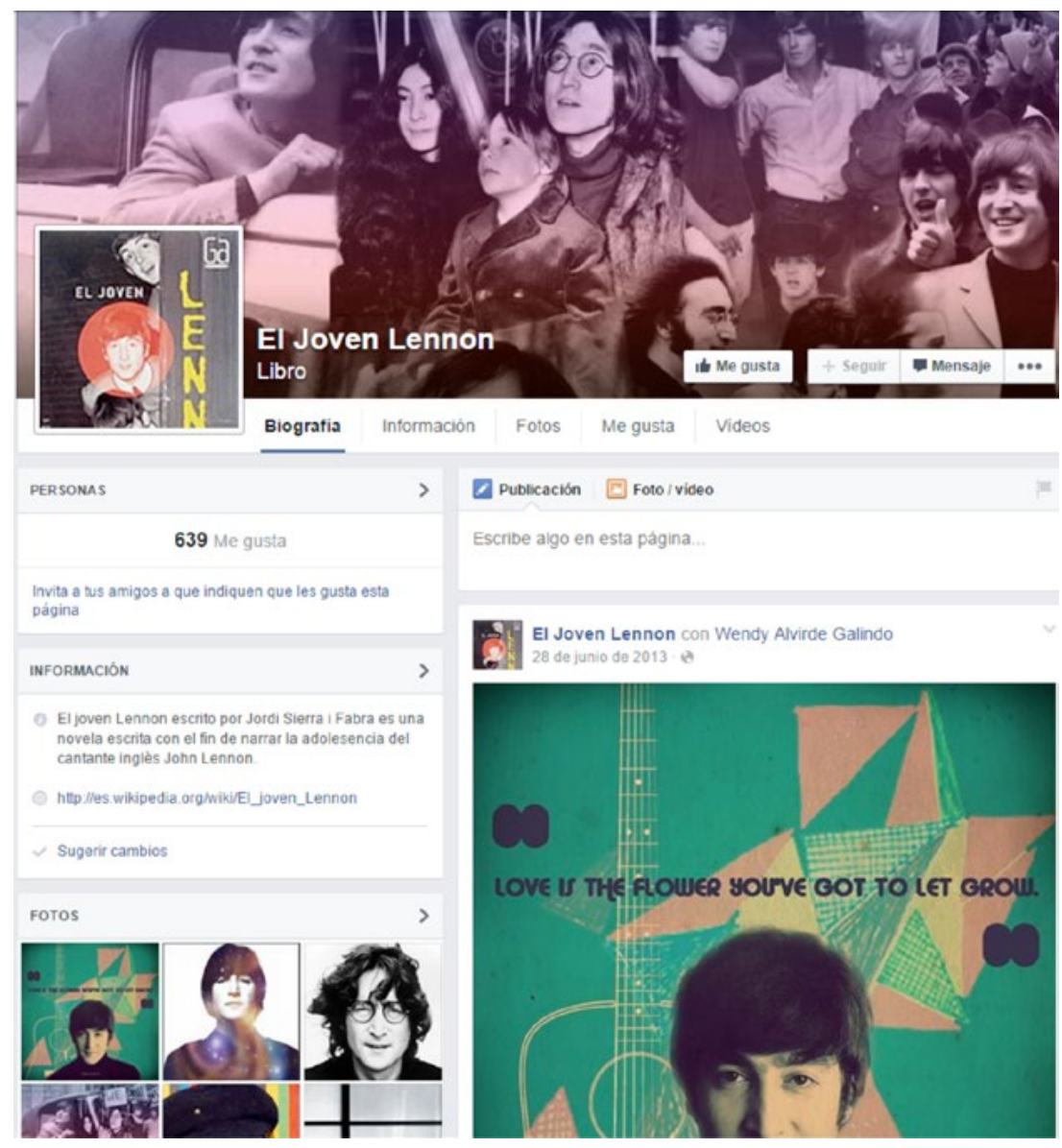

Página en Facebook de El Joven Lennon

Por lo tanto, el contexto digital del que disponían los lectores de la obra era bastante precario. Para compensar estas carencias se desplegó un dispositivo de acompañamiento de los lectores con una triple vertiente, y en paralelo al proceso de lectura de la obra. Se habilitó un blog y un perfil de Tuenti con noticias del desarrollo de la investigación, concebidos también como punto de encuentro e información entre los participan-

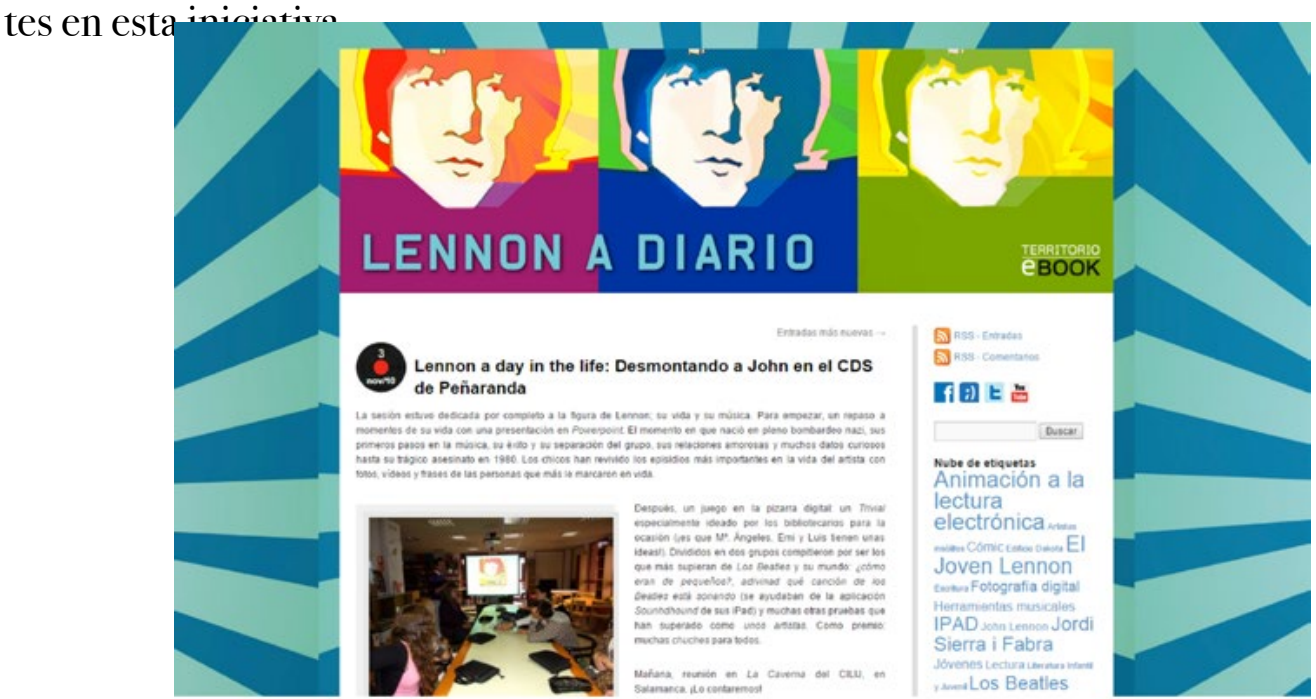

Blog Lennon a Diario 
Se desarrollaron un conjunto de actividades relacionadas con la vida y obra de Lennon (recreación de sus canciones a través de la Nintendo Wii, fotografías en Salamanca emulando la famosa de Abey Road, encuentro con Jordi Sierra i Fabra, etc.) y se articuló un sistema de dinamización de la obra a través de las intervenciones de personal especializado de la Fundación Germán Sánchez Ruipérez.

La investigación respondía a un diseño complejo en el que se habían de evaluar dos aspectos con apenas precedentes en la literatura científica: en qué medida los lectores realizan una adecuada apropiación de un dispositivo nuevo, no diseñado exclusivamente para la lectura, pero con funcionalidades específicas para ella, y de qué manera la biblioteca favorece tanto esta apropiación como la propia comprensión lectora de las obras a través de los procesos de dinamización emprendidos por la misma. Los resultados de la investigación pueden leerse en http://es.calameo.com/read/0005095631d2ce80a7ad1.

Uno de los aspectos más interesantes de este estudio tiene que ver con el uso del Ipad para la lectura. Los lectores aceptan el Ipad como dispositivo con una gran cantidad de posibilidades para el desarrollo de actividades de carácter recreativo, pero muestran resistencias a la incorporación del mismo en sus prácticas de lectura. ¿Significa esto que la lectura digital carece del atractivo ya demostrado en los dispositivos de tinta electrónica? ¿Qué el apego a los sistemas tradicionales para lecturas de carácter convencional es todavía intenso? En Quebec se desarrolló en 2013 un estudio sobre el uso del Ipad por parte de los estudiantes. En el mismo, se pueden leer los resultados de una encuesta realizada entre 6000 alumnos y 300 profesores sobre el uso de esta tableta en el medio educativo. Según el estudio los profesores encuentran entre sus principales ventajas las posibilidades de buscar información, la portabilidad, el desarrollo de una enseñanza más colaborativa, la adaptación al ritmo del alumno. Las ventajas percibidas por los alumnos se relacionan sobre todo con la accesibilidad a las redes y la organización del trabajo académico.

Una cuestión interesante que se plantea en este estudio es la relacionada con la lectura en los dispositivos móviles y concretamente en el Ipad. ¿Sirve para leer en las condiciones en que lo hacen habitualmente los estudiantes? Según este estudio, no. Según los resultados de la encuesta se produce una subutilización, un muy bajo uso, de libros electrónicos completos, algo que a priori constituiría una de las principales funciones de las tabletas. El estudio revela que menos del $3 \%$ de los alumnos indicaban haber leído libros completos en las pantallas del Ipad. De hecho, la lectura ocupa la última de las posiciones entre las actividades desarrolladas en la clase por parte de los alumnos, y entre las desarrolladas fuera de la clase ni siquiera aparece.

Investigaciones desarrolladas por el Centro Joan Ganz Cooney en 2012 y otras como las publicadas por el prestigioso Scientific American llegaban a conclusiones similares, afirmando, de una manera un tanto categórica, que el cerebro humano prefiere el papel. ¿Sería esto cierto? La hipótesis en las que se basan estas afirmaciones plantean que el cerebro humano percibe un texto en su totalidad, como una especie de paisaje fí- 
sico. Cuando leemos, construimos una representación mental del texto. Estas representaciones dejan una impronta que permite identificar con facilidad las topografías de un libro, las marcas de página y los signos más o menos indelebles que se perciben en ellos, dado que no existe una homogeneidad absoluta entre los ejemplares impresos, ni siquiera en los que forman parte de una misma tirada, sobre todo después de haber pasado por más de un lector.

Desde esta perspectiva el libro representa un medio caliente, parafraseando a Mac Luhann. La relación del lector con cada obra es una relación de identidad, en tanto que la diferencia físicamente del resto, de compromiso, en tanto que la obra, con su presencia representa una experiencia por venir, o una vivencia por recordar, cuando ya ha sido leída, y de inmersión en tanto el espacio de las páginas invita a la concentración y a la delectación.

La despersonalización de la literatura digital, en tanto que los elementos de reconocimiento permanecen ocultos y los de identificación y e inmersión quedan reducidos a un espacio homogéneo y uniforme, iría en detrimento de esta experiencia analógica, o caminaría por una senda completamente diferente.

A la luz de estos planteamientos, completamente generalizados en el momento en el que se desarrolla la investigación, no es extraño que la percepción de los lectores hacia la lectura en un dispositivo digital, además con pantalla retroiluminada, estuviera sujeto a la resistencia de quienes estaban habituados a otro tipo de lectura.

Desde este punto de vista la investigación fijó un referente, el de la apropiación de un dispositivo nuevo, con capacidades multimedia en el momento de su aparición, el de la postura y las percepciones de un grupo de lectores sobre un momento crítico en la historia de la lectura.

Sin duda esta constituye un hito en la historia de los estudios sobre la lectura digital, que obtendrá un mayor reconocimiento cuando se comiencen a contextualizar, a nivel nacional e internacional, todas las iniciativas llevadas a cabo en esos años.

En 2011 se desarrollan también otros dos proyectos asociados a Territorio Ebook: Ebookeando en la Nieve y Lectores en la Nube.

\section{Ebookeando en la nieve.}

En este caso se trata de un proyecto dirigido al grupo de lectores comprendido entre los 40 y los 55 años, que han de leer El Manuscrito de Nieve de un autor que ya había participado en la primera experiencia de Territorio Ebook, Luis García Jambrina. En esta nueva fase se cuenta con un grupo de 40 lectores, pertenecientes a club de lectura, con los cuales se trabaja en un modelo de comprensión lectora más profundo que el desarrollado con los mayores de 55 años, buscando una mayor implicación y participación en el blog que se puso en marcha con motivo del desarrollo del proyecto. Además del blog el estudio acompañado de actividades paralelas con el fin de reforzar las acciones empren- 
didas a través de la dinamización de la lectura.

Estudiar esta franja de edad es sumamente interesante pues se encuentra en "terreno de nadie", en lo que a la lectura digital se refiere. Ocupan una franja intermedia en las estadísticas sobre prácticas digitales y su inclinación por una tendencia u otra reviste perfiles bastante inciertos.

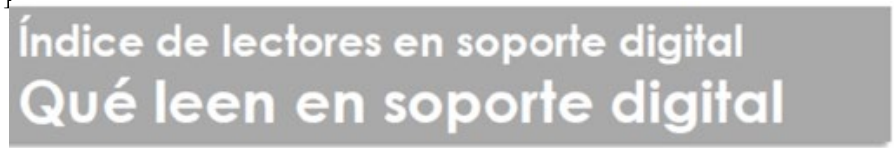

Base: Población de 14 ó más años. (12.761)

\section{\% LECTORES EN SOPORTE DIGITAL Respuesta mútiple}

\begin{tabular}{|c|c|c|c|c|c|c|}
\hline \% Horizontales & $\begin{array}{l}\text { Total Lectores } \\
\text { en soporte } \\
\text { digital }\end{array}$ & $\begin{array}{c}\text { Webs, Blogs, } \\
\text { Foros,... }\end{array}$ & Prensa & Revistas & Libros & Cómics \\
\hline & $\%$ & \% & $\%$ & $\%$ & $\%$ & $\%$ \\
\hline TOTAL (12.762) & 52,7 & 42,4 & 34,9 & 7,4 & 6,8 & 3,1 \\
\hline \multicolumn{7}{|l|}{ SEXO } \\
\hline Hombre (6.331) & 59,9 & 48,2 & 43,7 & 9,1 & 7,2 & 4,6 \\
\hline Mujer (6.430) & 45,6 & 36,8 & 26,2 & 5,7 & 6,4 & 1,6 \\
\hline \multicolumn{7}{|l|}{ EDAD } \\
\hline De 14 a 24 años (1.773) & 85,3 & 75,0 & 44,9 & 14,0 & 13,7 & 7,4 \\
\hline De 25 a 34 años (2.405) & 78,1 & 67,6 & 51,1 & 11,5 & 10,3 & 6,0 \\
\hline De 35 a 45 años $(2.466)$ & 60,1 & 46,3 & 44,0 & 8.2 & 7,3 & 2,8 \\
\hline De 45 a 54 años (2.123) & 49,0 & 38,2 & 35,0 & 4,9 & 4,4 & 1,2 \\
\hline De 55 a 64 años (1.752) & 32,5 & 20,8 & 23,9 & 5,3 & 4,9 & 1,0 \\
\hline 65 años y mos (2.242) & 10,6 & 6,2 & 8,1 & 0,8 & 0,6 & - \\
\hline
\end{tabular}

Lectores en soporte digital. Fuente: Hábitos de compra y lectura de libros.

Federación de Gremios de Editores

Como se puede apreciar en la tabla superior la lectura digital no llega al 50\% entre los lectores comprendidos en esta franja de edad. Sus pautas de lectura se centran en la web y en la prensa digital. Son los que menos libros leen en este soporte, si exceptuamos a los mayores de 65 años. Además, su lectura se desarrolla mayoritariamente a través del ordenador.

Por lo tanto, el trabajo con ellos constituiría una muestra excelente para comprobar si las tareas de dinamización e intervención de la biblioteca ejerce una influencia positiva en lectores cuya inclinación natural, por formación, tradición y expectativas, no se encuentra en el ámbito digital.

Los lectores de este grupo, se comenta en las conclusiones del estudio, parece que no están tan acostumbrados al uso de la tecnología como podrían pensar antes de comenzar la experiencia. Leyeron en un modelo más avanzado que los mayores de 55 años, y sin embargo se sintieron menos satisfechos. Esperaban más de lo que han encontrado en el 
dispositivo, y llegaron cargados de muchos más prejuicios y resistencias que los mayores, no les basta como a ellos con el acompañamiento. Están llenos de dudas, ser o no ser lector digital, esa es la cuestión. Son lectores en tránsito, en tránsito permanente; un tránsito que abarca no solo su faceta de lector, sino toda su vida. Saben que no pueden cerrar los ojos a la tecnología, pero no es fácil perder lo que Marc Prensky llama el acento del inmigrante, y menos cuando se trata de "traicionar" al libro de papel. No obstante, a alguno el arrastre de este acento no les impide transitar entre el papel y el libro electrónico, soltando poco a poco el lastre de no poder oler o tocar los libros.

Una investigación que, como las precedentes, se articula sobre un complejo entramado en el que la lectura concita toda una serie de intervenciones en torno a los lectores para crear un espacio de participación y reflexión sobre la obra y en el que la tecnología adquiere un protagonismo fundamental. Se trata de comprobar cuál es la naturaleza de las barreras que esta puede imponer, como se vencen las resistencias hacia la misma y como se profundiza en la comprensión de la obra.

En febrero de 2011, cuando comenzaba la investigación de la FGSR, en la que el Ipad había sido elegido como soporte de lectura, dos instituciones americanas, iModerate Research Technologies y Brock Associates, planteaban las posibilidades que las tabletas tenían para la lectura, algo que ya se había demostrado en las investigaciones que se estaban desarrollando en ese momento en la Fundación Germán Sánchez Ruipérez.

En febrero de 2011 también, la International Publishing Association (IPA) lanza su estudio sobre el estado del libro digital en el mundo, alertando sobre sus posibilidades a los editores y miembros de la cadena del libro.

\section{Un giro y un cambio metodológico determinante}

Todos los proyectos desarrollados hasta este momento habían compartido una premisa importante, muy asentada en los programas de promoción y estímulo de la lectura y asumidas como axiomas en los desarrollos de políticas bibliotecarias de todo el mundo: las actividades de acompañamiento de la lectura, como presentaciones, encuentros con autores, firmas de libros, representaciones y lecturas de contenidos etc. redundan positivamente en el comportamiento de público lector, constituyendo por si mismas un estímulo, un acicate, para el desarrollo de hábitos lectores, y un fortalecimiento de la voluntad de practica de esta actividad. El desarrollo de conferencias, visitas, blog, por otra parte, no es algo privativo del ámbito bibliotecario, sino que se extiende a la idiosincrasia de todo el sector del libro.

El problema surge cuando se constata que aunque se percibe cierto impacto positivo en los niveles de comprensión del grupo experimental respecto al grupo de control, en las diferentes fases de los proyectos, se comprueba que estos procedimientos, de carácter tradicional, no aprovechan adecuadamente todas las posibilidades que la tecno- 
logía permite, que la dinamización externa a la obra constituye una buena estrategia de acompañamiento, pero que reviste un carácter más superficial de cara a la comprensión de las obras.

Este planteamiento no aparece en ninguna de las investigaciones que se habían desarrollado sobre prácticas lectoras anteriormente. Los elementos contextuales, los paratextos lectores se habían considerado siempre como elementos de valor añadido, dotados de sentido por y para el libro. Pero ¿Qué ocurre si se comprueba que cursos, conferencias, entrevistas, talleres, sitios web y otros patrones de intervención clásica no revisten la efectividad prevista? Evidentemente, las consecuencias son determinantes para la propia concepción de la lectura como un fenómeno complejo que requiere un grado de atención mucho más especializado que el articulado en torno a los programas de animación tradicional. Y los planteamientos metodológicos de una investigación que pretende desentrañar los resortes estimulan la participación, la empatía y la comprensión de los lectores, habían de corregir radicalmente las premisas sobre las que estaban asentados. ¿Invalida esto los resultados anteriores? En modo alguno, antes bien fundamenta el funcionamiento de estrategias vinculadas con la dinámica tecnológica y demuestra que esta obedece a lógicas que no se pueden extraer directamente del entorno analógico. Esto no significa una exclusión taxativa de los procedimientos anteriores, pero si una relativización y supeditación a estrategias que abordan lo digital desde el propio sistema.

La hipótesis que se desarrolla a partir de entonces, y que se postula en las siguientes investigaciones, lectores en la Nube y Nube de Lágrimas, es que el enriquecimiento y la dinamización de las obras ha de efectuarse desde el propio contenido de las mismas, con carácter interno, por una parte. Por otra se decide que la deconstrucción de la obra ha de ser un paso previo a la dinamización de la misma. Son dos decisiones importantes, pues la primera determina la necesaria articulación de los contenidos según una lógica estrictamente digital, lo que apunta a la necesaria colaboración entre editores y bibliotecarios, y la segunda a la necesidad de desarrollar análisis en profundidad de los elementos estructurales de la obra para conectarlos con las actividades desarrolladas por los profesionales de la dinamización.

\section{Lectores en la nube y Nube de Lágrimas}

Partiendo de estas premisas, en septiembre de 2011 y en febrero de 2013 se ponen en marcha las dos siguientes fases del Proyecto Territorio Ebook: Lectores en la nube y Nube de Lágrimas. En este caso dirigidos, el primero al sector de edad comprendido entre los 19 y los 39 años, y el segundo a todos los sectores de edad. Una vez investigados los sistemas de apropiación de los dispositivos, tanto de tinta electrónica como Ipad, el comportamiento de los lectores hacia las obras con el acompañamiento de la biblioteca, la dinamización operativa en contextos temáticos y demográficos diferentes ¿Qué quedaba por investigar sobre la lectura digital? En este caso se plantea la necesidad de avanzar 
un paso más en las investigaciones y se analiza la construcción del lector social, esto es, en qué medida las redes sociales pueden contribuir a la implicación de los lectores en el desarrollo de una obra y en la dinamización de la misma.

Para ello se eligió el trabajo con las dos principales redes sociales generales existentes, Twitter y Facebook. En el primer caso se eligió una obra de Lorenzo Silva, Niños Feroces, para su dinamización a través de Twitter, en el segundo, el de Nube de Lágrimas, una obra de Rosa Montero, Lagrimas en la Lluvia. En esta segunda investigación además se emplearon herramientas de lectura social específica como Readmill. De nuevo era la primera vez que se abordaba un fenómeno emergente por parte de la FGSR para someterlo a estudio. La lectura en relación con las redes sociales no había estado sujeta a investigación alguna en el mundo con la perspectiva de análisis del impacto sobre la comprensión que se abordaba ahora.

Un ejemplo del impacto de las redes sociales sobre la lectura lo representa Facebook. El 3 de enero de 2015 Mark Zuckerberg, uno de los creadores de esta red, lanzo un club de lectura bajo con el reto de leer un libro cada dos semanas y comentarlo con aquellos que se apuntaran a su club de lectura en Facebook. La página A year of books alcanzó en pocas horas cientos de miles de adhesiones.

El fenómeno no es extraño y constituye una importante evidencia de la importancia que pueden revestir las redes sociales en el ecosistema del libro. Lo singular del fenómeno es que casi todos los acontecimientos señalados han sido movimientos posteriores a las investigaciones pioneras desarrolladas por la Fundación Germán Sánchez Ruipérez sobre este fenómeno, cuyas interesantes conclusiones y desarrollo pueden leerse en los informes respectivos:

http://es.calameo.com/read/000509563b29f8e098c8b?authid=1Hep0aZXdV9e http://es.calameo.com/read/00050956329f4617050ec

\section{Repercusiones de la investigación desarrollada por la FGSR.}

Desde que en 2009 comenzara el desarrollo del programa Territorio Ebook la Fundación ha diseñado una etnografía de la lectura digital en España, sin precedentes. Cerca de medio millar de lectores, entre 9 y 75 años han pasado por las diferentes fases del proyecto. Se han analizado los sistemas de apropiación de dispositivos, se han diseñado programas de formación, amparados por los resultados de las investigaciones, se han propiciado espacios de conversación entre bibliotecas y editores, como los encuentros celebrados anualmente en Peñaranda entre ambos colectivos (Conversaciones Líquidas), se ha trabajado con equipos de investigación de varias universidades, se han establecido tipologías de lectores y estrategias de acompañamiento para cada una de las mismas, se han profundizado en el uso de las redes sociales y la lectura y se ha concluido con el desarrollo de una spin off, Nubeteca, www.nubeteca.info con el diseño de una propuesta integral de gestión de la colección y la lectura digital en la biblioteca pública. Un programa 
que carece de antecedentes en el ámbito mundial.

Las investigaciones desarrolladas por la FGSR han demostrado que las prácticas de lectura se ven considerablemente alteradas por la aparición de nuevos soportes y nuevos formatos, conformando discursos y realidades completamente nuevas. Que el libro electrónico, en convergencia con el fenómeno de expansión de Internet y la proliferación de dispositivos móviles (portátil, teléfono móvil, PDA’s, miniconsola y, en menor medida, el e-reader) en la vida cotidiana de los ciudadanos de países económicamente desarrollados, han empezado a transfigurar la figura del lector, sus hábitos de lectura y escritura, abriendo nuevos puentes para que se manifiesten formas genuinas en la manera en cómo se conciben, producen, leen, utilizan, intercambian, distribuyen y reelaboran los textos escritos. Esta «tercera revolución del libro» no sólo ha provocado una gran incertidumbre en el sector editorial, sino que también es motivo de preocupación entre investigadores y agentes culturales, por las consecuencias que pudieran tener en los hábitos lectores las mutaciones que implica la lectura multimodal así como las nuevas formas acceder al vasto universo de contenidos vertidos en Internet.

Frente a este fenómeno han surgido mensajes, en cierto modo reticentes al nuevo modelo. Varios neurólogos de reconocido prestigio defienden en sus estudios que el cerebro humano modifica su comportamiento debido al uso de las nuevas tecnologías. Según estos expertos, nuestro cerebro experimenta reacciones diversas para asumir el ritmo constante y la intensidad de estímulos cerebrales que comporta el consumo de cualquier tipo de contenidos culturales a través de los diferentes tipos de pantallas.

Maryanne Wolf (2010) sostiene que lo que hace nuestro cerebro para aprender a leer, es una actividad antinatural. Establece nuevas conexiones entre estructuras y circuitos dedicados originalmente a otros procesos cerebrales más básicos, como la visión y el habla. Nuestro cerebro sería un ejemplo de arquitectura abierta, en el sentido que lo emplean los informáticos para referirse a un sistema lo bastante versátil para cambiar o reorganizarse a fin de adaptarse a las demandas variables que recibe. Según Wolf no leemos porque cambie nuestro cerebro primitivo, sino que, leyendo, el cerebro continúa cambiando, tanto psicológica como intelectualmente. Sostiene la autora. Gary Small (2009) explora cómo la marcha imparable de la tecnología está modificando la forma en la que se desarrollan las mentes jóvenes y cómo perciben e interpretan la información de su entorno. Describe el cambio producido por el avance tecnológico a nivel de construcciones mentales y sus consecuencias para el futuro: ¿Cuáles son los impactos profesionales, sociales y políticos de esta modificación de algunas funciones de nuestro cerebro? ¿Cómo podemos adaptarnos? Aunque la inmersión de alta tecnología podría mejorar algunos procesos de aprendizaje y facilitar el desarrollo de mentes creativas, también tiene sus riesgos, entre ellos el mayor aislamiento social y las "nuevas adicciones", hiperactividad y déficit de atención. Una línea de pensamiento compartida por William Powers, Richard Stallman o el ya mencionado Nicholas Carr.

Por su parte, Thierry Baccino (Baccino et. al., 2015), sostiene que la lectura sobre pantalla exige un mayor nivel de intervención del cerebro e incluso un comportamiento 
diferente. Según este autor, las zonas del encéfalo que controlan la toma de decisiones y los razonamientos complejos se activan con más intensidad que cuando se efectúa la lectura en papel. Debido a la necesidad de efectuar más elecciones en la consecución de la lectura, a la existencia de más información disponible, a la superposición de elementos con sistemas de codificación y de interpretación diferente, el nivel de complejidad es mayor y por lo tanto el peligro de pérdidas de significado también. Sin embargo, la lectura en pantalla se constituirá en hábito, mantiene Baccino, y el papel correrá el riesgo de provocar la misma sensación que las películas en blanco y negro: se tendrá la impresión desagradable de la regresión. Baccino ha desarrollado interesantes experiencias de oculometría para detectar los parámetros de calidad lectora en los ámbitos analógico y digital, diferenciando la percepción (de los signos, de las palabras), de la comprensión. Esta línea de investigación representa una de las líneas de investigación más interesantes desde el momento en el que el medio digital difumina la frontera entre canal y representación determinando la aparición de todo tipo de escrituras líquidas. Mendelsund (2015) ha demostrado la singular relación existente entre lectura y compresión según las representaciones y contextos en que esta se desarrolla. "La historia de nuestra lectura, dice el autor, es siempre la de un recuerdo. Cuando leemos, estamos absortos. Y, cuanto más absortos estamos, menos capaces somos de utilizar nuestra mente para analizar la experiencia en la que nos hallamos inmersos. Así pues, cuando hablamos de la sensación de la lectura estamos hablando en realidad del recuerdo de haber leído. Y ese recuerdo de la lectura es un falso recuerdo"

Los estudios más próximos a las experiencias desarrolladas por la Fundación Germán Sánchez Ruipérez son los impulsados por un grupo de investigadores de la Universidad de Stavanger, Noruega, liderados por Anne Mangen. Una de sus últimas investigaciones (Mangen, 2014a), según la cual la lectura digital afecta profundamente a la comprensión de los textos, recibió un eco inusitado en todo tipo de medios que replicaron las conclusiones del mismo en su vertiente más adversa, simplificada, y tergiversada, esto es que la lectura de libros impresos mejora la comprensión de los textos. En este estudio para determinar la capacidad de comprensión de los lectores para el papel y los dispositivos electrónicos, los investigadores noruegos compararon la lectura de ambos formatos. Para ello agruparon a 50 voluntarios, quienes debieron leer un cuento corto (28 páginas) de la escritora estadounidense de misterios, Elizabeth George.

El grupo se dividió en 2, la mitad de ellos leyendo en formato papel y la otra en dispositivos electrónicos. Una vez terminada la lectura, ambos equipos debieron responder un test acerca de las características y personajes de la historia. Los investigadores concluyeron que quienes leyeron la versión impresa obtuvieron mejores resultados. Anne Mangen, investigadora principal del estudio, ya había desarrollado un estudio anterior, comparando la lectura en papel y en un Ipad, en el que se encontraron diferencias entre ambas. Los lectores en papel obtuvieron mejores resultados en las medidas relacionadas con la empatía, la inmersión y la coherencia narrativa.

Para el equipo, "la retroalimentación háptica”, es decir, el sentido del tacto re- 
lacionado con el iPad, no ofrece el mismo soporte mental para la reconstrucción de una historia como la que ofrece un libro de bolsillo" (Mangen, 2014b). Otros estudios de la autora muestran una postura más prudente (Mangen, 2014c), en la medida en que reconoce que la línea divisoria, en relación con la atención y la comprensión lectora, no es tan categórica entre los diferentes soportes

Lo que la Fundación ha demostrado con sus investigaciones es que la apropiación del dispositivo y la formación en lectura digital constituyen dos factores imprescindibles para el desarrollo de la misma. Cualquier investigación que desconozca estas circunstancias llegará a conclusiones erróneas.

En los comentarios desarrollados más arriba sobre algunas de las conclusiones del proyecto Beatlepad se hacía alusión a las preferencias de los usuarios hacia la lectura en papel, y las resistencias al uso del Ipad para esa actividad. Unos años más tarde, cuando esa cuestión se plantea con los lectores que han empleado el Ipad para la lectura de Lágrimas en la Lluvia, los resultados son completamente diferentes.

Cuando se les pregunta sobre su impresión de la lectura en pantalla, de todas las respuestas la mejor puntuada, con 6.47, es la más interesante pues uno de los argumentos más utilizados por los detractores de la lectura digital es precisamente el de la dificultad de concentración en la lectura y la imposibilidad de seguir argumentaciones con la misma facilidad con que se hace en el papel. En este caso los lectores no han experimentado ningún tipo de dificultad y han valorado especialmente este aspecto como muy positivo. 3.2 La lectura en la pantalla te ha resultado

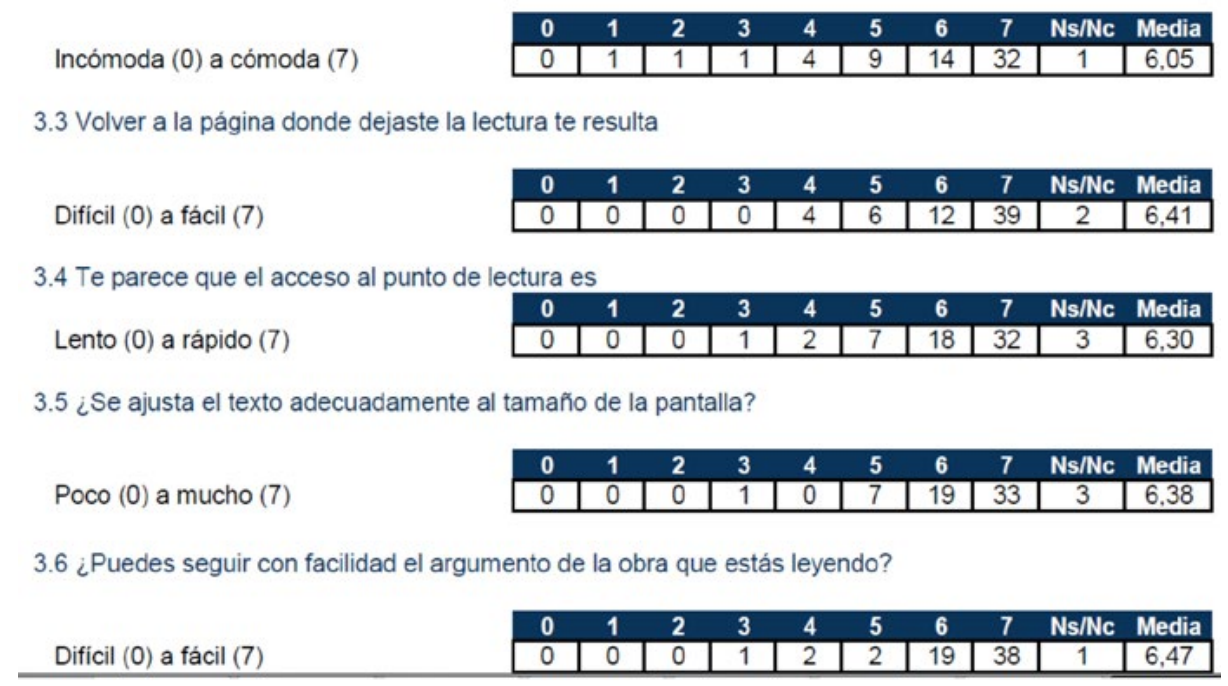

Un hecho significativo es que, cuando se interroga a los lectores sobre el soporte preferido para la lectura de los distintos géneros textuales, la respuesta mayoritaria es en el Ipad, excepto para Poesía y Teatro. 
Poesía

Teatro

Novela

Prensa y revistas

Libros informativos

Informes

Otros

\begin{tabular}{|c|c|c|}
\hline Papel & iPad & Ns/Nc \\
\hline 40 & 15 & 8 \\
\hline 36 & 19 & 8 \\
\hline 24 & 38 & 1 \\
\hline 17 & 45 & 1 \\
\hline 15 & 45 & 3 \\
\hline 21 & 40 & 2 \\
\hline 12 & 25 & 26 \\
\hline
\end{tabular}

Esta respuesta, descontextualizada podría plantear dudas sobre el sentido de la migración de un soporte a otro, y las razones de sus preferencias, aunque estas se han podido colegir por algunas de las respuestas anteriores, donde valoraban las prestaciones del Ipad. Por ello se les interrogó acerca de la influencia del medio de lectura en la misma, esto es, si consideraban que el dispositivo influye de alguna manera en la experiencia de lectura o esta es independiente del mismo. La respuesta mayoritaria incide en la argumentación anterior, como puede comprobarse.

6.7 Consideras que la experiencia de lectura es independiente del soporte de la obra.

Para ti es igual leer en papel que en el iPad

Sí, es igual, lo que me interesa es la obra

No, realmente me parecen muy interesantes y

utilizo las posibilidades que ofrece la lectura en el iPad

No, no es igual pero no sabría decir por qué

No, me gusta más leer en el iPad
$16 \quad 25,40 \%$

$34 \quad 53,97 \%$

$8 \quad 12,70 \%$

$5 \quad 7,94 \%$

La constatación redunda en el hecho de que son las prestaciones complementarias las que favorecen y diferencian un tipo de lectura distinto que la del papel, mejorando la misma.

Cuando se interroga a los lectores para que argumenten sus impresiones sobre su experiencia de lectura en el Ipad, en relación con la lectura en papel, los razonamientos refuerzan la hipótesis de que la socialización y la colaboración son elementos consustanciales con la lectura digital. 
- Que leo mucho más

- Al tener internet tengo más facilidad para entretenerme y desconcentrarme de la lectura

- Creo que el estar conectado con internet enriquece mucho la lectura

- Me sigue gustando el papel, pero esto es más cómodo

- Poder hacer comentarios facilita el carácter interactivo de este tipo de lectura

- Que leo más rápido.

- La mayor de las diferencias es que llevas la luz incorporada y puedes leer donde y cuando quieras sin molestar a nadie ( ejm cama)

- Me es mas cómodo leer fuera de casa con el libro electrónico.

- "El no tener que leer en un mamotreto (en libro en papel) ha sido muy satisfactorio. En un principio participé en el proyecto porque me llamaba la atención pero mi idea era seguir leyendo en papel. Después de la experiencia he comprobado que es una maravilla leer en iPad por la luz (iluminación), el tamaño de la letra, la comodidad, el poco peso, el poder ir a enlaces enriquecidos desde el propio libro, poder conectarte a Facebook a la vez... Tener todo en uno es incomparable."

- Que hay una manera más rápida de compartir la lectura y los pensamientos.

- Que es una manera distinta, para mi más fría si el libro que estás leyendo lo haces solo para ti, pero si la lectura es compartida, es estupendo de esta forma.

Son muchas las manifestaciones de los lectores que abundan en estos argumentos, insistiendo en la experiencia de lectura, en la movilidad que proporciona, y en el resto de prestaciones que entraña el uso de un dispositivo móvil.

En general los lectores han valorado muy positivamente la experiencia de lectura desarrollada a través del Ipad, algo especialmente significativo teniendo en cuenta que todos eran miembros de diferentes clubes de lectura que hasta esa experiencia habían leído en papel.

¿Qué es lo que ha cambiado respecto a la investigación anterior? En primer lugar el giro metodológico al que se hacía alusión más arriba. La compenetración del lector con la obra digital, la profundización en los contenidos y la intervención sobre los mismos ha de estar presidida por el desarrollo de dinámicas internas al texto, no externas a él. Por otra parte, se llevó a cabo una profunda integración de la formación, en el uso del dispositivo y en el uso de las aplicaciones (Facebook y Readmill), con la dinamización. Finalmente, los elementos de identificación física que ejercen de isobaras en el libro físico, se pueden compensar con la personalización y la socialización en el libro digital. Anotaciones, subrayados, comentarios, hilos de conversación, ejercen esa tarea de fijación que permite solventar la distancia emocional y perceptiva inherente a las obras digitales. A partir de Nube de Lágrimas, la investigación sobre la lectura habrá de operar con estos parámetros. 


\section{Referencias Bibliográficas}

- Alonso Arévalo, J., y Cordón García, J. A. (2015). El libro como sistema: Hacia un nuevo concepto de libro. Cuadernos De Documentación Multimedia, 26. http://revistas.ucm.es/ index.php/CDMU/article/view/50628

- Alonso Arévalo, J., Gómez Díaz, R., y Cordón García, J. A. (2015). Ebooks en bibliotecas: Gestión, tratamiento y aplicaciones. Buenos Aires: Alfagrama.

- Baccino, T., et. al. (2015). The impact of paper-based versus computerized presentation on text comprehension and memorization. Computer in Human Behaviour, 54

- Carr, N. (2010). Superficiales, ¿qué está haciendo internet con nuestras mentes? Madrid: Taurus.

- Cordón García, J. A., Gómez Díaz, R., y Alonso Arévalo, J. (2011). Gutenberg 2.0: La revolución de los libros electrónicos. Gijón: Trea.

- Chartier, R. (2006). Qué es un texto. Madrid: Circulo de Bellas Artes.

- Federación de Gremios de Editores de España: 2011. Hábitos de lectura y compra de libros. Madrid: 2012.

- Karsenti, T., \& Fievez, A. (2013). L'iPad à l'école: Usages, avantages et défis. Montreal: CRIFPE.http://karsenti.ca/ipad/rapport_iPad_Karsenti-Fievez_FR.pdf

- Mangen, A. et. al. (2014a). Mystery story reading in pocket print book and on kindle: Possible impact on chronological events memory. The International Society for the Empirical Study of Literature and Media, Turin, Italy July 21-25

- Mangen, A., \& Kuiken, D. (2014b). Lost in an iPad: Narrative engagement on paper and tablet. Scientific Study of Literature, 4(2), 150-177.

- McKenzie, D. F. (2005). Bibliografía y sociología de los textos. Madrid: Akal.

- Mendelsund, P. (2015). Qué vemos cuando leemos: Una fenomenología con ilustraciones. Barcelona: Seix Barral.

- Observatorio de la Lectura y el Libro. (2016). El libro digital: Aproximación a la realidad de las editoriales presentes en LIBER 2015. Madrid: Ministerio de Cultura. http:/ / ow.ly/ YW8qS

- Powers, W. (2010). Hamlet's blackberry: A practical philosophy for building a good life in the digital age. HarperCollins, 2010. 
- Sanchez, E., et. al. (2011). Ebook 55+: Los lectores mayores de 55 años y los libros electrónicos Fundación Germán Sánchez Ruipérez. http://es.calameo.com/ $\mathrm{read} / 00050956333174 \mathrm{ff} 88 \mathrm{~d} 82$

- Small, G. (2009). Elcerebro digital: Cómo las nuevas tecnologías están cambiando nuestra mente. Barcelona: Urano.

- Stallman, R. (2011). The danger of e-books. http://stallman.org/articles/ebooks.pdf

- Stern, D. (2010). Ebooks: From institutional to consortial considerations. Online, 2936.

- The Cocktail Analysis. (2009). Prospección del mercado de dispositivos lectores de ebooks. The Cocktail Analysis.

- Tveit, A. K., \& Mangen, A. (2014). A joker in the class: Teenage reader's attitudes and preferences to reading on different devices. Library \& Information Science Research, 36 (3-4), 179-184.

- Wolf, M. (2010). Como aprendemos a leer. Barcelona: Ediciones B. 